Meta

Journal des tradlucteurs

Translators' Journal

\title{
Sigles et noms propres
}

\section{Dorothy Nakos}

Volume 35, numéro 2, juin 1990

URI : https://id.erudit.org/iderudit/003690ar

DOI : https://doi.org/10.7202/003690ar

Aller au sommaire du numéro

Éditeur(s)

Les Presses de l'Université de Montréal

ISSN

0026-0452 (imprimé)

1492-1421 (numérique)

Découvrir la revue

Citer cet article

Nakos, D. (1990). Sigles et noms propres. Meta, 35(2), 407-413.

https://doi.org/10.7202/003690ar

Ce document est protégé par la loi sur le droit d'auteur. L’utilisation des services d'Érudit (y compris la reproduction) est assujettie à sa politique d'utilisation que vous pouvez consulter en ligne.

https://apropos.erudit.org/fr/usagers/politique-dutilisation/
Cet article est diffusé et préservé par Érudit.

Érudit est un consortium interuniversitaire sans but lucratif composé de l’Université de Montréal, l'Université Laval et l'Université du Québec à Montréal. Il a pour mission la promotion et la valorisation de la recherche. https://www.erudit.org/fr/ 


\section{SIGLES ET NOMS PROPRES ${ }^{1}$}

\section{INTRODUCTION}

Le but de notre exposé est d'étudier les relations qui existent entre le sigle et le nom propre. Nous définissons, dans un premier temps, le sigle de la façon suivante: nom (unité lexicale) formé d'initiales et de syllabes provenant a) d'un mot (lexie simple), par exemple «T» pour «Titus» à l'époque romaine, b) d'un mot composé (lexie construite à partir d'au moins deux composants), par exemple «ECG» pour «électrocardiogramme», ou c) d'un groupe de mots (lexie complexe), par exemple «CIA» pour «Central Intelligence Agency». Comme nous le savons déjà, le sigle se prononce soit lettre à lettre, par exemple «ECG» et «CIA», soit comme un mot, par exemple «UNESCO» ou «FNAC», plus difficile à prononcer; dans ce dernier cas, il devient acronyme ou sigle acronymique. Comme tout autre nom, le sigle, qu'il soit acronymique ou non acronymique, peut s'intégrer au discours.

Notons également que le sigle peut, dans certains cas, être international (ex. «UNESCO»), ce qui en principe le rend assimilable au nom propre (ex. «Sigmund Freud» demeure «Sigmund Freud»), mais par ailleurs, il peut revêtir une forme différente dans d'autres langues (ex. «NATO» en anglais correspond à «OTAN» en français), ce qui se produit également le plus souvent pour le nom commun (ex. «dog» correspond à «chien», «door» à «porte»). De plus, un même sigle peut présenter des variantes graphiques, s'écrivant à l'intérieur d'une même langue tantôt avec des majuscules et des points, tantôt avec des minuscules sans points et ce, quelle qu'en soit la prononciation : lettre à lettre ou comme un mot (ex. «O.T.A.N.» et «OTAN», «cellule Apud» et «APUD cell», «Apud» désignant «amine precursor uptake and decarboxylation»). En ce sens, la graphie variable du sigle peut paraitre inattendue dans l'ensemble du lexique (comme peuvent l'être également les lettres majuscules initiales du nom propre). Qu'en déduire sinon qu'au premier abord, le sigle formerait une catégorie intermédiaire entre le nom propre et le nom commun? Notre propos ici est de montrer les rapports qu'entretiennent nom propre et sigle; autrement dit, l'association de noms communs peut donner naissance à un sigle qui est un nom propre (ex. «CISO») et inversement, l'association de mots où figurent un ou plusieurs noms propres peut donner naissance à un sigle qui est soit un nom commun (ex. «VWF» pour «von Willebrand factor») soit un nom propre (ex. «P.U.F.» pour «Presses universitaires de France»).

Rappelons, dans un deuxième temps, que le sigle est de plus en plus utilisé dans toutes les formes de communications: manchettes de journaux, ouvrages didactiques, bulletins techniques et autres (par exemple, dans Electron Microscope Techniques 1987, 6/2, p. 207: «Three-Dimensional Reconstructions from Serial Micrographs using the IBM PC», article de Stephen J. Young et autres). D'ailleurs, les dictionnaires de sigles de plus en plus nombreux en font foi. Depuis la Seconde Guerre mondiale, les sigles sont devenus numériquement plus importants, ne serait-ce que par la présence d'organismes de plus en plus nombreux à désigner et la nécessité de recourir à un langage international 
simple et codifié dans le cas des nouvelles technologies (ex. «laser», «l'antigène AgHBs»). Certains auteurs comme Mitterrand (1981: 64) limitent l'emploi du sigle aux «locutions dénominatives utilisées pour la désignation officielle des grandes organisations [...]»; toutefois, dans ce dernier cas, l'auteur se contredit en reconnaissant l'existence de sigles dans les «jargons techniques» (1981: 67)

Toutefois, la siglaison n'est pas un phénomène récent. Comme nous l'avons vu, il existait déjà à l'époque romaine; les soldats romains portaient des insignes gravés des lettres SPQR pour «Senatus Populusque Romanus». Rappelons que N.S.J.C. représentait «Notre Seigneur Jésus-Christ» et I.N.R.I., «Jesus Nazarenus Rex Iudaeorum». Au XVII ${ }^{e}$ siècle, les Nouveaux Convertis étaient désignés par le sigle N.C. (qui a donné «néci» dans les Cévennes). Certains auteurs comme Calvet (1980: 9-18) et Gehenot (1975: 279282) ont étudié en détail l'historique du sigle. et sigle.

Nous allons, dans notre exposé, analyser trois rapports possibles entre nom propre

$1^{\circ} \quad$ Le sigle formé à l'origine de noms communs auxquels peut s'ajouter un nom propre devient un nom propre (cas des appellations officielles).

Sans vouloir prétendre à l'exhaustivité, ce qui serait pratiquement impossible à cause du nombre incroyable de sigles existants, notre analyse de deux ouvrages spécialisés (TOWELL et SHEPPARD, Acronyms, Initialisms and Abbreviations Dictionary et DUBOIS, Dictionnaire de sigles nationaux et internationaux) montre bien que dans cette première catégorie se retrouvent surtout les sigles désignant des organismes et des sociétés de toutes sortes. On relève également des mouvements politiques (SALT pour «Strategic Arms Limitation Talks» peut servir de qualificatif à «accord» pour donner «accord Salt», des titres de revues comme $J C B$ pour «Journal of Cell Biology» et PNAS de «Proceedings of the National Academy of Sciences of the United States of America», des langages informatiques comme COBOL («Common Business Oriented Language), FORTRAN («formulation transposée») et ALGOL («Algorithmic Language»), et bien d'autres encore.

Comme nous portons davantage notre attention sur l'aspect linguistique des sigles devenus noms propres (sigles propres), nous traiterons de certaines tendances observées. Dans le cas de sigles désignant des organismes nationaux (sigles indigènes), il existe rarement de correspondant dans une autre langue (par exemple, le sigle acronymique AFNOR pour «Association française de normalisation»). Toutefois, si le pays a deux langues officielles, comme c'est le cas au Canada, le sigle d'un organisme du Gouvernement fédéral a généralement un équivalent officiel dans l'autre langue (MRC pour «Medical Research Council» et CRM pour «Conseil de la Recherche Médicale»); ce n'est pas nécessairement le cas au niveau provincial (ex. OLF de «Office de la langue française» demeure OLF en anglais puisqu'il n'existe aucune indication ni aucune obligation à le traduire, bien au contraire). Il est évident que pour des raisons de clarté le traducteur devrait donner la signification du sigle indigène. En revanche, en ce qui a trait aux organismes internationaux, il existe parfois des sigles graphiquement différents selon la langue utilisée (ex. CEE de «Communauté économique européenne» est traduite par EEC, «European Economic Community») et parfois des sigles uniques reconnus dans plusieurs langues ayant pourtant des appellations officielles différentes dans chaque langue (ex. UNICEF dont l'appellation officielle est «United Nations International Children's Emergency Fund» en anglais et «Fonds international de Secours à l'enfance» en français).

Dans le cas des sociétés, ce sont les impératifs économiques qui priment. En effet, une société est identifiée dans le monde d'une seule façon (ex. IBM) à moins que la juridiction gouvernementale en ait décidé autrement. Par exemple, la société Bell Canada 
s'est transformée en holding, «les Entreprises Bell Canada Inc.» ou EBC en français, «Bell Canada Entreprises Inc.» ou BCE en anglais; ce holding comprend maintenant, en plus de Bell Canada, l'entreprise à Vancouver «BCE Development Corporation» qui a été traduit en français par «Corporation de développement $\mathrm{BCE}$ », «corporation de développement» étant un anglicisme (il s'agit en fait d'une société immobilière) et «BCE» une forme erronée dans ce contexte puisque EBC existe en français.

De plus, le sigle non acronymique ayant rarement plus de quatre lettres pour des raisons mnémotechniques entre autres, il en résulte inévitablement une prolifération d'homonymes sigliques (ex. CEC désigne au moins cinq sociétés différentes en Europe, dont «Carbonisation Entreprise Céramique»). Le sigle peut également s'intégrer à un syntagme pour donner, par exemple, fonds anti-NPD (NPD représentant le «Nouveau Parti démocratique» au Canada).

Les sigles acronymiques peuvent adopter diverses formes:

a) regroupement de lettres initiales de mots pleins comme dans CISO et ICOS;

b) regroupement de syllabes initiales comme dans SUNOCO de «Sun Oil Company» et ALCAN de «Aluminium Canada», ce dernier ayant l'avantage d'avoir non seulement l'appellation sous-jacente mais aussi le sigle communs à l'anglais et au français; s'il n'y avait pas eu dans ce cas de coïncidence de mots en anglais et en français, il est probable que l'appellation et le sigle seraient demeurés anglais;

c) regroupement de lettres et de syllabes donnant des sigles comme AFNOR déjà cité où - NOR représente «normalisation» (dans ce cas, aux lettres initiales s'est ajoutée une troncation syllabique);

d) interversion de lettres comme dans TERMIA pour «Association internationale de terminologie» et «International Association of Terminology»;

e) prononciation déterminant la graphie par le remplacement ou l'ajout de lettres comme dans ESSO «Standard Oil» (notons que Esso est connu internationalement sous le nom de la société mère «Exxon» qui n'a aucune signification particulière dans aucune langue) ou encore l'omission de toutes les lettres d'un des mots de l'appellation initiale comme dans SAJIB pour «Société d'animation du jardin et de l'institut botaniques de Montréal», le M n'étant pas représenté dans le sigle.

Nous avons également relevé le cas intéressant d'homonymie acronymique voulue, CISO et ICOS déjà cités, où une partie du signifié demeure fixe, à savoir «international» et «sciences onomastiques», mais où la lettre $C$ peut désigner aussi bien le «Centre», le «Congrès» que le «Comité». Nous sommes ici en présence d'une économie siglique puisque le contexte détermine le sens à donner à $C$.

En plus de correspondre à une appellation bien particulière, l'acronyme peut se colorer intentionnellement par l'ajout d'un message sous-jacent. Ce procédé existait déjà à l'époque romaine quand les chrétiens écrivaient tXò̀s (signifiant poisson) sur les murs

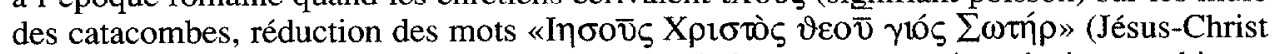
Fils du Dieu Sauveur). D'ailleurs l'acronyme était souvent représenté pictographiquement par le symbole du poisson. Les organismes connotés affectivement sont nombreux de nos jours. En anglais, nous avons par exemple ACT ( $\ll$ Action for Children in Trouble»), CARE ( $\ll$ Cooperative for American relief Everywhere»), SCARE ( $\ll$ Students Concerned About a Ravaged Environment»), SOS («Sanity on Sex»), MADD («Mothers Against Drunk-Driving») et SADD («Students Against Drunk-Driving») aux États-Unis. Au Québec sont nés des organismes comme AQTE («Association québécoise des techniques de l'eau») — un dessin paru dans Le Devoir du premier août 1988 montrait un homme en train d'arroser sa pelouse avec l'indication suivante : «Avec le boyau, allez-y mollo», ce qui tend à prouver qu'ici le sigle n'est pas simple coïncidence, AIDE («Association internationale pour le développement et l'équité» - en France, nous avons 
l'«Association pour l'information et pour la défense économique des débiteurs de tabac»), COQ ( «Club des ornithologues du Québec»), FRAP («Front révolutionnaire d'action politique») dans les années soixante-dix, FORCE («Fonds d'organisation et de coopération pour l'emploi»), RAJ («Regroupement autonome des jeunes»). En Europe, nous avons par exemple AMI («Association pour l'information médicale», «Assistance médicale intérim»), ATLAS pour «Assises de la traduction littéraire en Arles», Arles devenant alors la ville française de la traduction.

Il faut tout de même signaler que si la connotation paraît manifeste dans les cas cités, les sigles que nous venons de nommer servent aussi à désigner des organismes n'ayant pas de lien évident avec le nom commun évoqué ; par exemple ACT désigne également «Automatical Channel and Time (Toshiba Corporation)», CARE «Capitol Reef National Monument», AMI «Alliance des magasins indépendants». Nous pourrions aussi croire que des sigles comme FIL ou AVAL désigneraient des organismes dont les activités seraient liées aux mots évoqués; il n'en est rien car FIL désigne, dans les ouvrages consultés, «Fédération internationale de la laiterie» et AVAL «Association pour la vente dans l'alimentation». Notons en terminant cette partie que les homonymes sigliques représentent divers organismes, sociétés et autres, non seulement dans un même pays, mais aussi dans des pays différents utilisant ou non la même langue.

$2^{\circ} \quad$ Le nom propre se retrouve dans la formation de sigles qui sont des noms propres (cas des appellations patronymiques ou toponymiques).

Nous rangeons dans cette catégorie les sigles servant à désigner des noms de personnages célèbres ou des noms de lieux. De nouveau, l'Antiquité fournit déjà des exemples de cette utilisation: $T$, déjà cité, désignait «Titus», $M$ «Marcus». Nous avons relevé des exemples de cet usage en France (BB pour «Brigitte Bardot», VGE pour «Valéry Giscard d'Estaing» et JJSS pour «Jean-Jacques Servan-Schreiber»), mais cet usage ne semble pas aussi répandu qu'en anglais américain, la présence d'un «middle name» expliquant en partie cette réduction; par exemple, FDR pour «Franklin Delano Roosevelt», LBJ pour «Lyndon Baines Johnson», JFK pour «John Fitzgerald Kennedy». Au Canada, nous avons PET pour «Pierre Elliott Trudeau». Le traducteur doit tenir compte des considérations culturelles avant d'adopter tel quel un sigle.

La réduction en sigles acronymiques ou non acronymiques s'applique également aux toponymes construits d'au moins deux mots comme U.K. («United Kingdom»), G.B. («Great Britain») ou G.-B. («Grande-Bretagne»), U.S.A. ou parfois E.U. en français, URSS («Union des Républiques socialistes soviétiques») et USSR en anglais, Benelux («Belgique, Nederland, Luxembourg»). Nous avons des cas de villes et de quartiers comme dans N.-D.-G. pour «Notre-Dame-de-Grâce» à Montréal.

Dans les deux parties que nous venons de traiter, certains sigles propres résultant de l'association de noms communs ou de noms propres peuvent également, grâce à la lexicalisation, donner lieu à des dérivés nominaux ou adjectivaux comme «néci» déjà cité, «péquiste» (de P.Q., «parti québécois»), «cégétiste» (de CGT, «Confédération générale du travail»), «puciste» (de P.U.C., «Paris Université Club»), «onusien» (ONU, «Organisation des Nations Unies»), «bénéluxisation».

$3^{\circ} \quad$ Le nom propre sert à la formation de sigles qui sont des noms communs (cas du vocabulaire technique et scientifique).

\section{A) DOMAINES TECHNIQUES}

La maniabilité des sigles ainsi que le peu d'espace qu'ils occupent sur les appareils concourent à expliquer la présence de très nombreux sigles dans les domaines techniques visant l'application des connaissances humaines. Contrairement aux deux catégories précédentes où le nom propre entrait souvent dans la formation de sigles désignant des 
appellations patronymiques et toponymiques ainsi que des appellations officielles, si l'on compare l'ensemble des sigles se rapportant aux domaines techniques, seul un nombre très restreint de sigles techniques font intervenir le nom propre. On doit également noter que la première catégorie des appellations officielles sert à qualifier certains termes du vocabulaire technique; par exemple, «API gravity», échelle utilisée par l'American Petroleum Institute, «ASTM cetane number (of a diesel fuel)», «ASTM supercharge ratings» où ASTM représente l'organisme de normalisation «American Standards For Testing and Materials». Dans ces trois cas, l'appellation officielle siglée résultant de la réduction d'une lexie complexe devient à son tour un mot servant à qualifier un substantif, le tout formant un syntagme ayant un sens bien particulier. Le cas inverse existe également, c'est-à-dire le nom de la société est écrit au long, le sigle n'existant pas, mais le produit de la société est siglé, par exemple «Philips EM 400» où EM désigne «electron microscope».

De plus, une lettre initiale provenant d'un nom propre peut servir à former des séries paradigmatiques. Par exemple, dans le cas de certaines charges, nous relevons HB, HK (sigle qui résulte d'une réduction d'un autre sigle $\mathrm{KHN}$ ), HR, HV où $\mathrm{H}$ représente «hardness number», les autres lettres des noms propres: «Brinell», «Knoop», «Rockwell», «Vickers»; d'où HB signifie «Brinell hardness number», HK ou KHN, «Knoop hardness number», etc. Nous avons également $\mathrm{HSc}$ où $\mathrm{Sc}$ représente «Scleroscope», nom de fabrique d'un instrument. Ce procédé, par sa simplicité, demeure évidemment intéressant pour le fabricant; notons également que seule la première lettre initiale du syntagme «hardness number» a été retenue dans tous les cas sauf dans celui où nous avons le synonyme KHN à côté de HK. Le syntagme simple «hardness number» se trouve alors qualifié par l'ajout d'un nom propre.

\section{B) DOMAINES SCIENTIFIQUES}

Quant aux domaines scientifiques touchant les connaissances humaines, il est certain que les syntagmes longs et complexes favorisent la formation de sigles. En outre, en médecine plus particulièrement, plus le résultat est significatif dans l'application (notamment sous forme de diagnostic ou de thérapeutique) et plus le nom est utilisé, plus il y a de chances que se forme un sigle. Par exemple, le bacille de Koch (BK) et le bacille bilié de Calmette-Guérin (BCG) ont été importants dans le cas de la tuberculose, le virus d'Epstein-Barr (EBV ou virus EB) du groupe herpès virus, important pour la compréhension de toute une série de tumeurs et de la mononucléose infectieuse. Les exemples relevés à la suite d'un dépouillement systématique prouvent que les scientifiques privilégient l'emploi d'un sigle unique pour désigner la même notion en anglais et en français. Autrement dit, BCG, par exemple, demeure en français et en anglais, WISC également, même si à l'origine il provient du syntagme anglais «Wechsler Intelligence Scale for Children». C'est aussi le cas de WAIS («Wechsler Adult Intelligence Scale»), WBS («Wechsler-Bellevue Scale»), WMS («Wechsler Memory Scale»). Dans le cas que nous venons de citer, le patronyme «Wechsler» ainsi que le «S» du nom commun «scale» signifiant échelle servent de dénominateur commun pour former une série paradigmatique. Plusieurs cas peuvent se présenter:

a) Le sigle résulte de la réduction d'un groupe de mots (lexie complexe) formé d'un mot clé et de qualificatifs toponymiques ou patronymiques (cas des éponymes).

Voici quelques exemples: $\mathrm{BCG}$ et $\mathrm{EBV}$ déjà cités, $\mathrm{BCG}$ donnant le dérivé «bécégite», accident dû à l'inoculation de BCG, BAL («British-Anti-Lewisite») pour désigner une substance capable de neutraliser la lewisite, gaz de guerre arsenical, VWF («von Willebrand factor») déjà cité, qui est une protéine du plasma sanguin entrant dans la 
coagulation, RS, («Ringer solution»). De même, l'encéphalite est spécifiée dans les cas suivants par l'ajout d'un toponyme: VEE («Venezuela Equine Encephalitis»), CEE («Central Europe Encephalitis»), MVE («Murray Valley Encephalitis»), SLE («St. Louis Encephalitis»: il s'agit de St-Louis dans l'Illinois), WEE («Western Equine Encephalitis»). La série paradigmatique est ici due à la lettre $\mathrm{E}$, constante dans chaque cas pour désigner des encéphalites virales, le toponyme signifiant le lieu d'origine de la maladie; de plus, le sigle peut servir à nommer le virus, par exemple «virus SLE».

Il existe aussi un certain nombre de cas où le sigle anglais ne pénètre pas le vocabulaire français, par exemple NPD en anglais se rend en français par le syntagme «maladie de Niemann-Pick» et GTO se rend par «organe neurotendineux de Golgi».

b) À l'intérieur d'un groupe de mots (lexie complexe), le mot clé est écrit au long et les qualificatifs toponymiques ou patronymiques sont siglés.

Citons les exemples suivants: «fièvre Q» (Q désigne «Queensland» en Australie), «test au BCG», «thérapie par la BCG» ou encore «BCG test» et «BCG thérapie», ces deux derniers devenant alors des syntagmes nominaux, «facteur LL» (facteur Laki-Lorand) appelé aussi «fibrinase» ou «facteur III (de la coagulation)», «bande H» pour la «strie de Hensen» dans le muscle strié, «syndrome de CREST» où CREST est une réduction extrême puisque chaque lettre de l'acronyme représente une lexie complexe ou une lexie construite de plusieurs composants $(\mathrm{C}=$ «calcinose sous-cutanée», $\mathrm{R}=$ «syndrome de Raynaud», $\mathrm{E}=$ «dysfonction de l'œsophage», $\mathrm{S}=$ «sclérodactylie», $\mathrm{T}=$ «télangiectasies»).

Mentionnons aussi que la nomenclature scientifique (noms d'animaux, de plantes ou de minéraux) que nous ne traitons pas en détail ici est également riche en sigles qui s'associent à un nom commun pour former un syntagme commun, par exemple le Macacus Rhesus, qui est une catégorie de singe, se réduit en «Rh» pour donner «facteur $R h$ », «antigène $R h$ », «agglutinogène $R H$ », qui est un type d'antigène. On a même des «fotus $R h+»$, des «facteur $R h+»$ et «facteur $R h-»$.

c) À l'intérieur d'un groupe de mots (lexie complexe), le mot clé est siglé et le qualificatif topononymique ou patronyme est écrit au long.

On trouve «TAP-Schiff» pour «réaction au tétraacétate de plomb Schiff» (réaction utilisée par les histochimistes il y a quelques décennies) donnant lieu à des lexies complexes comme «substance» ou «matériel de TAP-Schiff positif», "réaction TAP-Schiff positive». À noter également la formation mixte d'un symbole chimique et d'un nom propre comme dans «réaction $\mathrm{O}_{2}$-Schiff» $\left(\mathrm{O}_{2}\right.$ signifiant oxygène).

d) Le sigle-symbole résulte de la réduction d'un patronyme (cas des unités de mesure).

Dans le cadre de notre étude, certaines unités de mesure siglées seraient en fait des symboles d'usage international dérivant d'un patronyme auquel pourrait s'ajouter un composant comme «méga», «déci», «milli», «kilo». Citons, par exemple, A (Angström), $\mathrm{Bq}$ (Becquerel), C (Celsius), Ci (de Pierre et Marie Curie), F (Faraday), Hz (Hertz), J (Joule), V (Volt), W (Watt), S (Siemens), K (Kelvin), N (Newton), R (Roentgen), S ou S.f. (Svedberg), Pa (Pascal) pouvant donner par exemple KPa pour KiloPascal.

Nous observons dans cette partie les possibilités multiples de formation de sigles communs à partir d'un ou de plusieurs noms propres (ex. BJ déjà cité) visant à raccourcir des syntagmes descriptifs souvent longs et complexes. De fait, syntagmation et siglaison vont de pair, le second étant l'aboutissement logique du premier. Citons à ce sujet Kocourek (1982: 121): «[...] l'utilité des sigles et des acronymes est due au fait qu'ils 
combinent dans la communication spécialisée, la maniabilité syntagmatique d'un seul mot avec le caractère sémantique descriptif du syntagme sous-jacent étendu».

Nous pouvons ajouter qu'il existe un grand nombre de combinaisons possibles de sigles et de noms communs (ex. «fièvre $Q$ » déjà cité) ou de sigles et de noms propres (ex. TAP-Schiff cité et U.V. Schiff où U.V. signifie ultra-violet) ou encore des deux à la fois (ex. «réaction TAP-Schiff positive» cité) où le sigle n'est plus le résultat d'un syntagme mais «l'origine» d'un nouveau syntagme. En ce sens, le sigle se comporte exactement comme tout autre nom commun qui devient l'élément clé d'un syntagme figé et monosémique.

\section{CONCLUSION}

En conclusion, nous pouvons dire que si le sigle n'a pas au point de départ de valeur descriptive comme c'est aussi généralement le cas du nom propre, néanmoins il a la particularité de faire partie intégrante de l'ensemble du lexique, du vocabulaire technique et scientifique et des désignations officielles. Il dérive d'abord d'un syntagme nominal donnant lieu à toutes sortes de possibilités, agissant comme nom et souvent comme composant; il peut avoir un rôle d'adjectivation en s'opposant à un autre nom (ex. «virus SLE» cité) et donner naissance à des dérivés nominaux (ex. «bécégite» cité) ou adjectivaux (ex. étude «onusienne»). Sa graphie, comme nous l'avons vu, tient tantôt du nom propre, tantôt du nom commun. De fait, nous avons essayé de montrer que le sigle peut être à la fois nom propre et nom commun en fonction de son utilisation précise dans le discours.

DOROTHY NAKOS

Université Laval, Québec, Canada

\section{NOTE}

1. Texte augmenté d'une conférence donnée le 18 août 1987 à Québec au Congrès international des Sciences onomastiques.

\section{BIBLIOGRAPHIE}

CALVET, L.-J. (1980), Les sigles, Paris, P.U.F., coll. Que sais-je? 1811, 123 p.

Compilation of ASTM Standard Definitions, Philadelphie, ASTM, 1986, 6 éd., $903 \mathrm{p}$.

CROWLEY, E.T. et R.C. THOMAS (1975), Acronyms and Initialisms Dictionary, Détroit, Gale Research Company, $4^{\mathrm{e}}$ éd., $635 \mathrm{p}$.

Dictionnaire des termes nouveaux des sciences et des techniques, Paris, CILF, 1983, $605 \mathrm{p}$

DUBOIS, M. (1977), Dictionnaire de sigles nationaux et internationaux, Paris, La maison du dictionnaire, $405 \mathrm{p}$

GARNIER, M. et V. DELAMARE (1985), Dictionnaire des termes techniques de médecine, Paris, Maloin, $21^{\mathrm{e}}$ éd., $873 \mathrm{p}$.

GEHENOT, D. (1975), «Le sigle : Aperçu linguistique» dans META 20/4, 27 1-307.

KOCOUREK, R. (1982), La langue française de la technique et de la science, Wiesbaden, Oscar Bandstetter Verlag GMBH-Co.Kg, $259 \mathrm{p}$.

Le Devoir, 1987, LXVIII/175 et LXXIII/181.

MAILLOT, J. (1981), La traduction scientifique et technique, Paris, Technique \& Documentation, $2^{\mathrm{e}}$ éd., 264 p.

MILLER, B.F. et C. BRACKMAN KEANE (1987), Encyclopedia and Dictionary of Medicine, Nursing and Allied Health, Philadelphie, Saunders Co., 1425 p.

MITTERRAND, H. (1981), Les mots français, Paris, P.U.F., coll. Que sais-je? 270, 6éd., 127 p.

TOWELL, J.E. et M.E. SHEPPARD (1987), Acronyms, Initialisms and Abbreviations Dictionary, Détroit, Gale Research Company, 6 vol. 3 index. 